REVISTA MATEMÁTICA de la

Universidad Complutense de Madrid

Volumen 9, número 2: 1996

http://dx.doi.org/10.5209/rev_REMA.1996.v9.n2.17573

\title{
Estabilidad orbital de satélites estacionarios
}

\author{
André DEPRIT y Teodoro LÓPEZ MORATALLA*

\section{Resumen}

Se establecen las condiciones, para un satélite alrededor de un planeta en rotación alrededor de su eje de inercia de mayor momento, bajo las que puede aparecer, en un sistema de referencia fijo en el planeta, dos posiciones de equilibrio con exponentes característicos imaginarios puros. En este caso, después de la apropiada normalización mediante una transformación de Lie, efectuada de forma mecánica con un procesador algebraico simbólico, se aplica el teorema de Arnold sobre formas cuadráticas no definidas. Se concluye que los equilibrios son estables en el sentido de Liapunov. Las condiciones de estabilidad se verifican en el caso de la Tierra.

\section{Abstract.}

For a satellite about an oblate planet in rotation about its axis of greatest inertia, conditions are given under which there may appear, in a frame fixed in the planet, two positions of equilibria with characteristic exponents that are purely imaginary. In which case, after appropriate normalization by Lie transformation executed mechanically through a symbolic algebraic processor, the theorem of Arnold about non definite quadratic forms is applied. It is concluded that the equilibria are stable in the sense of Liapunov. The conditions for stability are verified in the case of the earth.

1991 Mathematics Subject Classification: 68Q40, 70-04, 70F15, 70H15, $70 \mathrm{~J} 25$

1985 A. C. M. Classification: 1.1.4

Servicio Publicaciones Univ. Complutense. Madrid, 1996.

*Financiado parcialmente por el Ministerio Español de Educación y Ciencia, Proyecto DGICYT \# PB93-1236-C02-02. 


\section{Introducción}

Consideramos un satélite bajo la acción del potencial gravitatorio de la Tierra, con zonales y teserales hasta grado y orden 2 . Con este modelo de potencial estamos teniendo en cuenta la parte más significativa del potencial terrestre, en relación con satélites estacionarios. Con respecto a un sistema de referencia solidario al planeta, es bien conocido que existen cuatro posiciones de equilibrio en las cuales el satélite es estacionario. Estas posiciones se encuentran en los semiejes de la sección ecuatorial del elipsoide de inercia; dos de ellas son equilibrios linealmente estables, mientras que las otras son inestables (ver Blitzer et al. [2], Musen y Bailie [12] o Morando [11]).

Mediante un teorema de Arnold [1], demostraremos que los equilibrios linealmente estables son también estables en el sentido de Liapunov. Esto implica la existencia de un tonel en el espacio fásico que aisla el equilibrio; la órbita de cualquier punto dentro del tonel permanece en él, y la órbita de cualquier punto fuera del tonel nunca llega a penetrar en él.

Para cada equilibrio efectuamos una traslación del origen del espacio fásico al equilibrio, tomando como variables las variaciones $\zeta=$ $(\xi, \eta, \Xi, H)$ alrededor del equilibrio. El Hamiltoniano, que describe el movimiento en las proximidades del equilibrio puede desarrollarse como una serie

$$
\mathcal{H}=\sum_{n \geq 2} \mathcal{H}_{n}
$$

de potencias de $\xi, \eta, \Xi$ y $H$; los términos $\mathcal{H}_{n}$ son polinomios homogéneos de gradó $n$ en las variaciones. Existe una transformación completamente canónica $(\xi, \eta, \Xi, H) \longrightarrow(\phi, \psi, \Phi, \Psi)$ que reduce $\mathcal{H}_{2}$ a la forma

$$
\mathcal{H}_{2}=\omega_{1} \Phi-\omega_{2} \Psi
$$

dependiente sólo de los momentos. Los símbolos $\omega_{1}$ y $\omega_{2}$ son números reales estrictamente positivos. Por medio de una transformación de Lie, podemos extender la normalización hasta órdenes superiores (Deprit [4]). El resultado será un Hamiltoniano de la forma

$$
\mathcal{H}=\sum_{1 \leq k \leq n} \mathcal{H}_{2 k}(\Phi, \Psi)+\mathcal{P}^{(2 n+1)}(\phi, \psi, \Phi, \Psi)
$$


cuyos términos $\mathcal{H}_{2 k}$ son polinomios homogéneos de grado $k$ en $\Phi$ y $\Psi$, mientras que $\mathcal{P}^{(2 n+1)}$ es un desarrollo en serie de grado $2 n+1$, como mínimo. Dada la forma de $\mathcal{H}_{2}$, la linealización en el origen de las ecuaciones canónicas deducidas de $\mathcal{H}$ produce dos osciladores armónicos de frecuencias $\omega_{1}$ y $\omega_{2}$.

En el caso de la Tierra, $\mathcal{H}_{2}$ es una forma no definida. Por esta razón la estabilidad orbital no puede determinarse por el teorema de Liapunov. Pero, sin embargo, el teorema de Arnold puede aplicarse para determinar la estabilidad de los equilibrios. Una situación similar sucede con las posiciones triangulares de equilibrio del problema restringido de tres cuerpos. Allí, la forma cuadrática en el equilibrio es también no definida, pero Deprit y Deprit-Bartholomé [6] han usado el teorema de Arnold para establecer la estabilidad de los equilibrios. Nosotros seguimos la misma línea de argumentación.

El teorema de Arnold dice que el equilibrio es estable cuando un determinante $D_{4}$, deducido a partir de $\mathcal{H}_{4}$, es distinto de cero. En caso de que sea nulo, el teorema en sí mismo no determina la estabilidad. Meyer y Schmidt [10] completaron el teorema y probaron que el equilibrio es estable si existe algún $k(2 \leq k \leq n)$ tal que no se amula la cantidad $D_{2 k}=\mathcal{H}_{2 k}\left(\omega_{2}, \omega_{1}\right)$, obtenida haciendo $\Phi=\omega_{2}$ y $\Psi=\omega_{1}$.

La aplicación del teorema de Arnold está restringida a sistemas de dos grados de libertad. En sistemas de más de dos grados pueden producirse fenómenos de difusión a través del tonel. Por esta razón nos hemos centrado en el estudio de órbitas ecuatoriales.

Hemos tratado el problema analíticamente, no numéricamente, trasladando el razonamiento matemático a un lenguaje de programación científica. La traducción es posible mediante un sistema de software simbólico. En el apéndice presentamos un ejemplo del software empleado. Aplicamos nuestros programas a la Tierra, pero nos hemos asegurado de que son aplicables a otros planetas. 


\section{Equilibrios}

Desarrollamos el potencial $\mathcal{V}$ del planeta en la serie de armónicos esféricos

$\mathcal{V}=-\frac{\mu}{r}\left[1+\sum_{n \geq 1}\left(\frac{\oplus}{r}\right)^{n} \sum_{0 \leq m \leq n}\left(C_{n m} \cos m \lambda+S_{n m} \operatorname{sen} m \lambda\right) P_{n+m}(\operatorname{sen} \beta)\right]$,

siendo $\mu$ el producto de la masa de la Tierra por la constante de gravitación universal, $\oplus$ el radio ecuatorial del planeta y $P_{n m}$ las funciones asociadas de Legendre. Las coordenadas esféricas $r, \beta$ y $\lambda$ supónen que se ha adoptado un sistema de referencia ortonormal $B=\left(\boldsymbol{b}_{1}, \boldsymbol{b}_{2}, \boldsymbol{b}_{3}\right)$, solidario al planeta; en este sistema, $\beta$ es la latitud contada desde el plano engendrado por $b_{1}$ y $b_{2}$; la longitud $\lambda$ se mide en este plano a partir de $b_{1}$.

Habitualmente se usa el sistema de referencia geográfico en el que el origen es el centro de masas, $b_{3}$ es el eje de rotación y el plano engendrado por $b_{1}$ y $b_{3}$ es el plano meridiano que pasa por un punto arbitrariamente seleccionado (Greenwich en el caso de la Tierra). Por coincidir el origen con el centro de masas, los coeficientes $C_{10}, C_{11}$ y $S_{11}$ de $\mathcal{V}$ son nulos. La Geodinámica ha determinado modelos de potencial con diversos valores para $C_{n m}$ y $S_{n m}$. Nosotros usaremos los coeficientes del World Geodetic System publicado en 1984 (WGS-84).

En este artículo hemos adoptado un sistema de referencia diferente del geográfico, pero con el mismo origen. Es el definido por la base ortonormal $B^{\prime}=\left(b_{1}^{\prime}, b_{2}^{\prime}, b_{3}^{\prime}\right)$ en la dirección de los ejes principales de inercia del planeta, $\operatorname{con} b_{3}^{\prime}$ sobre el eje de momento de inercia mayor y con $b_{1}^{\prime}$ sobre el de momento menor. En adelante, para mayor sencillez en la notación, eliminaremos los apóstrofes de los $b_{i}^{\prime}$. En el sistema $B$, el potencial es de la forma

$\mathcal{V}=-\frac{\mu}{r}\left[1+\sum_{n \geq 2}\left(\frac{\oplus}{r}\right)^{n} \sum_{0 \leq m \leq n}\left(\Gamma_{n m} \cos m \lambda+\Delta_{n m} \operatorname{sen} m \lambda\right) P_{n m}(\operatorname{sen} \beta)\right]$.

Los productos de inercia en $B$ son nulos, por tanto $\Gamma_{21}=\Delta_{21}=\Delta_{22}=0$ (vér, por ejemplo, Heiskanen y Moritz [7]). No tendremos en cuenta la 
diferencia muy pequeña que existe entre $b_{3}$ y el eje de rotación; esto significa que tendremos

$$
\Gamma_{20} \simeq C_{20} \quad \text { y } \quad \Gamma_{22} \simeq \sqrt{C_{22}^{2}+S_{22}^{2}} .
$$

Para el análisis que sigue, es importante notar que $\Gamma_{20}$ es $\leq 0$ para un planeta en rotación alrededor del eje principal de mayor momento.

Consideramos el caso de un satélite en el campo del potencial $\mathcal{V}$. Sea $\boldsymbol{x}$ su vector de posición; puesto que suponemos el satélite en el plano ecuatorial, $x$ se descompone en la suma

$$
\boldsymbol{x}=x \boldsymbol{b}_{1}+y \boldsymbol{b}_{2} .
$$

La velocidad del satélite en el sistema $B$ es $\dot{x} b_{1}+\dot{y} b_{2}$. Pero el sistema $B$ se mueve con relación a un sistema inercial con la velocidad angular $\boldsymbol{\omega}=\boldsymbol{\omega} \boldsymbol{b}_{3}$, la cual consideraremos constante. El período $2 \pi / \omega$ es igual a un "día" sidéreo para el planeta. Así, la velocidad del satélite con relación al sistema inercial es

$$
\dot{\boldsymbol{x}}=\dot{\boldsymbol{x}} \boldsymbol{b}_{1}+\dot{y} \boldsymbol{b}_{2}+\boldsymbol{\omega} \times \boldsymbol{x} .
$$

Entonces, para un satélite ecuatorial la Lagrangiana es la función

$$
\mathcal{L}(\boldsymbol{x}, \dot{\boldsymbol{x}})=\frac{1}{2}(\dot{\boldsymbol{x}} \cdot \dot{\boldsymbol{x}})-\mathcal{V} .
$$

El momento $\boldsymbol{X}$ se define a partir de $\mathcal{L}$ por la relación $\boldsymbol{X}=\partial \mathcal{L} / \partial \dot{\boldsymbol{x}}$. En consecuencia

$$
\boldsymbol{X}=(\dot{x}-\omega y) \boldsymbol{b}_{1}+(\dot{y}+\omega x) \boldsymbol{b}_{2}
$$

Denotamos $X=\dot{x}-\omega y$ e $Y=\dot{y}+\omega x$. Por medio de la transformación de Legendre pasamos de las variables $(\boldsymbol{x}, \dot{x})$ a las $(\boldsymbol{x}, \boldsymbol{X})$, con lo que obtenemos el Hamiltoniano

$$
\mathcal{H}=\dot{\boldsymbol{x}} \cdot \frac{\partial \mathcal{L}}{\partial \dot{\boldsymbol{x}}}-\mathcal{L}=\frac{1}{2}(\boldsymbol{X} \cdot \boldsymbol{X})-\boldsymbol{\omega} \cdot \boldsymbol{x} \times \boldsymbol{X}+\mathcal{V} .
$$

Todos los términos de grado 3 y superior se omitirán en (3). Con esta restricción, la expresión final de $\mathcal{H}$ es

$$
\mathcal{H}=\frac{1}{2}\left(X^{2}+Y^{2}\right)-\omega(x Y-y X)-\frac{\mu}{r}\left[1+\frac{\oplus^{2}}{r^{2}}\left(-\frac{1}{2} \Gamma_{20}+3 \Gamma_{22} \frac{x^{2}-y^{2}}{r^{2}}\right)\right],
$$


de donde las ecuaciones del movimiento en el sistema $B$ son

$$
\begin{aligned}
& \dot{x}=\frac{\partial \mathcal{H}}{\partial X}=X+\omega y, \\
& \dot{y}=\frac{\partial \mathcal{H}}{\partial Y}=Y-\omega x, \\
& \dot{X}=-\frac{\partial \mathcal{H}}{\partial x}=\omega Y-\mu \frac{x}{r^{3}}\left\{1-3 \frac{\oplus^{2}}{r^{2}}\left[\frac{1}{2} \Gamma_{20}+\Gamma_{22}\left(2-5 \frac{x^{2}-y^{2}}{r^{2}}\right)\right]\right\}, \\
& \dot{Y}=-\frac{\partial \mathcal{H}}{\partial y}=-\omega X-\mu \frac{y}{r^{3}}\left\{1-3 \frac{\oplus^{2}}{r^{2}}\left[\frac{1}{2} \Gamma_{20}-\Gamma_{22}\left(2+5 \frac{x^{2}-y^{2}}{r^{2}}\right)\right]\right\}
\end{aligned}
$$

Si $\Gamma_{22}=0$, todos los puntos del círculo $x^{2}+y^{2}=r_{1}^{2}$, en el que el radio $r_{1}$ es la solución de la ectuación

$$
r^{5}-r_{0}^{3} r^{2}+\frac{3}{2} \Gamma_{20} \oplus^{2} r_{0}^{3}=0
$$

son equilibrios. (La distancia $r_{0}$ es tal que $\omega^{2} r_{0}^{3}=\mu$.) Pero, si $\Gamma_{22} \neq 0$, todos estos equilibrios desaparecen salvo cuatro puntos. En efecto, en este caso, hay dos, y sólo dos, posibilidades de anulación de las ecuaciones (5); éstas son $x=0$ e $y=0$.

Cuando $y=0$ (i.e., $r=x$ ) debe ser $X=0$ e $Y=\omega x$ para que se anulen $\dot{x}$ e $\dot{y}$. Esto implica también la anulación de $\dot{Y}$. La ecuación para $\dot{X}$, que se reduce a

$$
P(r)=r^{5}-r_{0}^{3} r^{2}-r_{0}^{3} \oplus^{2} \varepsilon_{1}=0,
$$

da la distancia entre el centro del planeta y el equilibrio. El parámetro $\varepsilon_{1}$ es la función

$$
\varepsilon_{1}=\frac{3}{2}\left(6 \Gamma_{22}-\Gamma_{20}\right)
$$

Este parámetro es positivo; por tanto, de acuerdo con el teroema de Descartes, la ecuación (6) tiene una, y sólo una, raíz positiva, $r_{1}$.

Por ser la ecuación (6) de quinto grado la solución no puede expresarse en términos simbólicos como una fórmula exacta. Por tanto buscaremos una aproximación. Para $\varepsilon_{1}=0$, la raíz $r_{1}$ es igual a $r_{0}$, y la derivada de $P$ con respecto a $\epsilon_{1}$ no es cero; entonces, de acuerdo con el Teorema de la Función Implícita, la raíz $r_{1}$, que emana de $r_{0}$, puede desarrollarse en serie de potencias de $\varepsilon_{1}$. Usando el método general propuesto por Deprit et al. [5] para resolver ecuaciones implícitas, 
encontramos que la expresión es

$$
\frac{r_{1}}{r_{0}}=1+\frac{1}{3} \varepsilon_{1}\left(\frac{\oplus}{r_{0}}\right)^{2}-\frac{1}{3} \varepsilon_{1}^{2}\left(\frac{\oplus}{r_{0}}\right)^{4}+\frac{44}{81} \varepsilon_{1}^{3}\left(\frac{\oplus}{r_{0}}\right)^{6}-\frac{260}{243} \varepsilon_{1}^{4}\left(\frac{\oplus}{r_{0}}\right)^{8}+\mathcal{O}\left(\varepsilon_{1}^{5}\right) .
$$

Designamos estos equilibrios como los puntos en el espacio fásico

$$
E_{1}=\left(r_{1}, 0,0, \omega r_{1}\right), \quad \text { y } \quad E_{3}=\left(-r_{1}, 0,0,-\omega r_{1}\right)
$$

Por otro lado, los equilibrios sobre el eje $y$ se obtienen de forma similar. Con $x=0$ (i.e., $r=y$ ) debe ser $X=-\omega y$ e $Y=0$ para que se anulen $\dot{x}$ e $\dot{y}$. La ecuación $\dot{Y}=0$ se reduce a

$$
r^{5}-r_{0}^{3} r^{2}-r_{0}^{3} \oplus^{2} \varepsilon_{2}=0,
$$

donde $\varepsilon_{2}$ es aquí el parámetro adimensional

$$
\varepsilon_{2}=-\frac{3}{2}\left(\Gamma_{20}+6 \Gamma_{22}\right)
$$

Al igual que con los equilibrios $E_{1}$ y $E_{3}$, para $\varepsilon_{2}>0$ existe una, y sólo una, raíz real $r_{2}>r_{0}>0$, que podemos aproximar mediante el desarrollo

$$
\frac{r_{2}}{r_{0}}=1+\frac{1}{3} \varepsilon_{2}\left(\frac{\oplus}{r_{0}}\right)^{2}-\frac{1}{3} \varepsilon_{2}^{2}\left(\frac{\oplus}{r_{0}}\right)^{4}+\frac{44}{81} \varepsilon_{2}^{3}\left(\frac{\oplus}{r_{0}}\right)^{6}-\frac{260}{243} \varepsilon_{2}^{4}\left(\frac{\oplus}{r_{0}}\right)^{8}+\mathcal{O}\left(\varepsilon_{2}^{5}\right) .
$$

Designamos estos equilibrios como los puntos en el espacio fásico

$$
E_{2}=\left(0, r_{2},-\omega r_{2}, 0\right), \quad \mathrm{y} \quad E_{4}=\left(0,-r_{2}, \omega r_{2}, 0\right) .
$$

Debemos observar, sin embargo, que $\varepsilon_{2}$ puede ser $<0$, es decir, cuando $\Gamma_{22}$ es $>-\frac{1}{6} \Gamma_{20}$. En este caso, de acuerdo con el teorema de Descartes, la ecuación (8) tiene 0 ó 2 raíces positivas. Estas situaciones han sido discutidas por Howard [8]. No las contemplamos aquí, porque ellas no ocurren para la Tierra.

\section{Estabilidad lineal}

Por razones de simetría es suficiente estudiar los equilibrios $E_{1}$ y $E_{2}$ en los ejes positivos. La estabilidad lineal en un equilibrio se deduce del 
sistema diferencial linealizado, planteando las ecuaciones variacionales en las proximidades de los equilibrios. Para ello efectuamos dos transformaciones completamente canónicas de las variables $(x, y, X, Y)$ a las $\zeta_{i}=\left(\xi_{i}, \eta_{i}, \Xi_{i}, H_{i}\right), i=1$ ó 2 , definidas por las ecuaciones

$$
x=\eta_{1}+r_{1}, \quad X=H_{1}, \quad y=-\xi_{1}, \quad Y=\omega r_{1}-\Xi_{1}
$$

para $E_{1}, \mathrm{y}$

$$
x=\xi_{2}, \quad X=\Xi_{2}-\omega r_{2}, \quad y=\eta_{2}+r_{2}, \quad Y=H_{2},
$$

para $E_{2}$. Los ejes $\eta$ así definidos van en la dirección radial, mientras que los $\xi$ van según la tangente, formando un sistema directo (ver Figura 1). Los Hamiltonianos transformados son respectivamente

$$
\begin{aligned}
\mathcal{H}^{(i)}= & -\frac{1}{2} \omega^{2} r_{i}^{2}+\frac{1}{2}\left(\Xi_{i}^{2}+H_{i}^{2}\right)-\omega\left(\xi_{i} H_{i}-\eta_{i} \Xi_{i}\right)-\omega^{2} r_{i} \eta_{i} \\
& -\frac{\mu}{\rho_{i}}\left\{1-\frac{\oplus^{2}}{\rho_{i}^{2}}\left[\frac{1}{2} \Gamma_{20}-(-1)^{i} 3 \Gamma_{22} \frac{\xi_{i}^{2}-\eta_{i}^{2}-2 r_{i} \eta_{i}-r_{i}^{2}}{\rho_{i}^{2}}\right]\right\},
\end{aligned}
$$

con la distancia al nuevo origen de coordenas definida por

$$
\rho_{i}=\sqrt{\xi_{i}^{2}+\left(\eta_{i}+r_{i}\right)^{2}} .
$$

Desarrollando las potencias inversas de $\rho_{i}$ en series de potencias de $\xi_{i}$ y $\eta_{i}$, podemos escalar los Hamiltonianos según el grado $p+q$ de los productos $\xi_{i}^{p} \eta_{i}^{q}$, con lo que los $\mathcal{H}^{(i)}$ quedan expresados por series de la forma

$$
\mathcal{H}^{(i)}=\mathcal{H}_{0}^{(i)}+\mathcal{H}_{1}^{(i)}+\mathcal{H}_{2}^{(i)}+\ldots+\mathcal{H}_{n}^{(i)}+\ldots
$$

El orden cero $\mathcal{H}_{0}^{(i)}$ es el valor del Hamiltoniano (4) en el equilibrio; entonces, es una constante, que podemos omitir en $\mathcal{H}^{(i)}$. Además, $\mathcal{H}_{1}^{(i)}$ es cero, por tratarse del desarrollo alrededor del equilibrio. El término $\mathcal{H}_{2}^{(i)}$ es una forma cuadrática en las variaciones $\xi_{i}, \eta_{i}, \Xi_{i}$ y $H_{i}$, y, para $n \geq 3, \mathcal{H}_{n}^{(i)}$ es un polinomio homogéneo de grado $n$ en $\xi_{i}$ y $\eta_{i}$.

La parte lineal del sistema diferencial se deduce del término $\mathcal{H}_{2}^{(i)}$. La expresión de $\mathcal{H}_{2}^{(i)}$ es

$$
\mathcal{H}_{2}^{(i)}=\frac{1}{2}\left(\Xi_{i}^{2}+H_{i}^{2}\right)-\omega\left(\xi_{i} H_{i}-\eta_{i} \Xi_{i}\right)+\frac{1}{2} \omega^{2}\left(\alpha_{i} \xi_{i}^{2}+\beta_{i} \eta_{i}^{2}\right)
$$




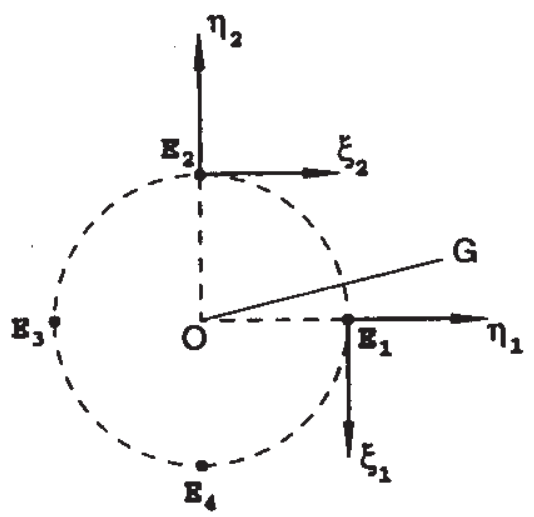

Figura 1: Equilibrios y ejes de los sistemas variacionales. La línea OG va en la dirección del meridiano de Greenwich.

con los parámetros estructurales

$$
\alpha_{i}=1-12(-1)^{i} \Gamma_{22} \frac{r_{0}^{3} \oplus^{2}}{r_{i}^{5}} \quad \text { y } \quad \beta_{i}=2\left(\frac{r_{0}^{3}}{r_{i}^{3}}-2\right)
$$

En notación matricial, el sistema variacional se presenta como

$$
\dot{\zeta}_{i}=A_{i} \zeta_{i}
$$

siendo $A_{i}$ la matriz

$$
A_{i}=\left(\begin{array}{cccc}
0 & \omega & 1 & 0 \\
-\omega & 0 & 0 & 1 \\
-\omega^{2} \alpha_{i} & 0 & 0 & \omega \\
0 & -\omega^{2} \beta_{i} & -\omega & 0
\end{array}\right)
$$

Los valores propios de $A_{i}$ son lás raíces de la ecuación característica

$$
\operatorname{det}\left(\lambda I-A_{i}\right)=\lambda^{4}+\omega^{2}\left(\alpha_{i}+\beta_{i}+2\right) \lambda^{2}+\omega^{4}\left(1-\alpha_{i}\right)\left(1-\beta_{i}\right)=0,
$$

y son de la forma $\pm i \omega_{i, 1}, \pm i \omega_{i, 2}$, con

$$
\begin{aligned}
& \omega_{i, 1}^{2}=\frac{\omega^{2}}{2}\left(\alpha_{i}+\beta_{i}+2+\sqrt{\left(\alpha_{i}-\beta_{i}\right)^{2}+8\left(\alpha_{i}+\beta_{i}\right)}\right) \\
& \omega_{i, 2}^{2}=\frac{\omega^{2}}{2}\left(\alpha_{i}+\beta_{i}+2-\sqrt{\left(\alpha_{i}-\beta_{i}\right)^{2}+8\left(\alpha_{i}+\beta_{i}\right)}\right) .
\end{aligned}
$$


Los equilibrios son linealmente estables si los valores propios son imaginarios puros, es decir, si las cantidades $\omega_{i, 1}$ y $\omega_{i, 2}$ son estrictamente positivas. Estas condiciones se verifican en el plano $\left(\alpha_{i}, \beta_{i}\right)$ en la región (I) donde $\alpha_{i}, \beta_{i}>1$, y en la región (II) donde $-3<\alpha_{i}, \beta_{i}<1, \quad 0<$ $\left(\alpha_{i}-\beta_{i}\right)^{2}+8\left(\alpha_{i}+\beta_{i}\right)$.

Para el equilibrio $E_{1}$ el punto $\left(\alpha_{1}, \beta_{1}\right)$ se encuentra fuera de las regiones (I) y (II). En efecto, $\alpha_{1}$ es $>1$ y $\beta_{1}$ es $<1$, por ser $\Gamma_{22}>0$ y $r_{1}>r_{0}$. Por tanto, para cualquier planeta que rote alrededor de su eje de mayor momento de inercia, el equilibrio $E_{1}$ y su simétrico $E_{3}$ son inestables.

Para los equilibrios $E_{2}$ y $E_{4}$ el estudio de la estabilidad lineal no es tan sencillo como en el caso precedente. Evidentemente, el punto $\left(\alpha_{2}, \beta_{2}\right)$ se encuentra en el cuadrante $\alpha_{2}, \beta_{2}<1$. Entonces, para determinar cuando el punto se encuentra en la región (II), es suficiente sustituir los parámetros $\alpha_{2}$ y $\beta_{2}$ en la ecuación de la parábola $\left(\alpha_{2}-\beta_{2}\right)^{2}+8\left(\alpha_{2}+\right.$ $\left.\beta_{2}\right)=0$ entre los puntos $(-3,1)$ y $(1,-3)$. En la práctica, la sustitución supone una cierta complejidad. Después de haber introducido la fracción $\bar{r}_{2}=r_{2} / r_{0}$, encontramos que las desigualdades

$$
\begin{aligned}
& \bar{r}_{2}<\sqrt[3]{2} \\
& \Gamma_{20}>\frac{1}{6}\left(\frac{r_{2}}{\oplus}\right)^{2}\left(10-31 \bar{r}_{2}^{3}+12 \sqrt{r_{2}^{3}\left(5 \bar{r}_{2}^{3}-2\right)}\right)
\end{aligned}
$$

son la condición necesaria y suficiente para que el punto $\left(\alpha_{2}, \beta_{2}\right)$ esté dentro de la región (II). Aparentemente (14) son condiciones para $r_{2}$. Pero, $r_{2}$ depende de $\Gamma_{20}$ y $\Gamma_{22}$ a través de la ecuación de quinto grado (8); por tanto (14) son, de hecho, condiciones para los coeficientes $\Gamma_{20}$ y $\Gamma_{22}$. Estas son aplicables a cualquier planeta.

De acuerdo con las definiciones (2), los valores

$$
\Gamma_{20}=-0.1082630 \times 10^{-2} \text { y } \Gamma_{22}=0.1814964 \times 10^{-5}
$$

para la Tierra verifican las condiciones (14). Por tanto, los equilibrios $E_{2}$ y $E_{4}$ en el caso de la Tierra son linealmente estables. 


\section{Estabilidad en el sentido de Liapunov}

Para aplicar el teorema de Arnold al equilibrio $E_{2}$, debemos normalizar el Hamiltoniano $\mathcal{H}^{(2)}$ de (11).

La normalización podemos efectuarla en variables ángulo-acción reales, pero es más sencillo hacerla en variables complejas, puesto que aquí tratamos con polinomios, mientras que con variables ángulo-acción debemos tratar con funciones trigonométricas. En lo que sigue eliminaremos los subíndices y superíndices $i$, en el entendido de que nos referimos exclusivamente al equilibrio $E_{2}$.

Como paso previo a la normalización del Hamiltoniano (11) debemos efectuar una transformación canónica al espacio complejo $w=$ $(u, v, U, V)$, tal que la parte cuadrática $\mathcal{H}_{2}$ adopte la forma normal compleja

$$
\mathcal{H}_{2}=i \omega_{1} u U+i \omega_{2} v V
$$

En las nuevas variables la matriz del sistema diferencial lineal deducido de (16), esto es, la transformada de la matriz $A$, debe ser diagonal con los valores propios $\pm i \omega_{1}, \pm i \omega_{2}$ en la diagonal principal. Puesto que $A$ es hamiltoniana, es $\operatorname{decir} A=J A^{\mathrm{T}} J,(J=$ matriz estándard simpléctica) y los cuatro valores propios son distintos, existe una transformación completamente canónica a una base formada por vectores propios, en la que matriz transformada de $A$ es precisamente de esta forma (Laub y Meyer [9]).

Procediendo como Deprit [3], encontramos que la transformación buscada es la transformación lineal $\zeta=B w$, cuya matriz asociada es

$$
B=\left(\begin{array}{cccc}
i a_{1} & -i a_{2} & a_{1} & a_{2} \\
-b_{1} & b_{2} & -i b_{1} & -i b_{2} \\
b_{1} \omega-a_{1} \omega_{1} & a_{2} \omega_{2}-b_{2} \omega & -i\left(a_{1} \omega_{1}-b_{1} \omega\right) & -i\left(a_{2} \omega_{2}-b_{2} \omega\right) \\
i\left(a_{1} \omega-b_{1} \omega_{1}\right) & -i\left(a_{2} \omega-b_{2} \omega_{2}\right) & a_{1} \omega-b_{1} \omega_{1} & a_{2} \omega-b_{2} \omega_{2}
\end{array}\right)
$$


donde los coeficientes $a_{1}, a_{2}, b_{1}, b_{2}$ están definidos por las igualdades

$$
\begin{array}{ll}
a_{1}^{2}=\frac{\omega_{1}^{2}+\omega^{2}(1-\beta)}{2 \overline{\omega_{1}\left(\omega_{1}^{2}-\omega_{2}^{2}\right)},}, & b_{1}^{2}=\frac{\omega_{1}^{2}+\omega^{2}(1-\alpha)}{2 \omega_{1}\left(\omega_{1}^{2}-\omega_{2}^{2}\right)} \\
a_{2}^{2}=\frac{\omega_{2}^{2}+\omega^{2}(1-\beta)}{2 \omega_{2}\left(\omega_{1}^{2}-\omega_{2}^{2}\right)}, & b_{1}^{2}=\frac{\omega_{2}^{2}+\omega^{2}(1-\alpha)}{2 \omega_{2}\left(\omega_{1}^{2}-\omega_{2}^{2}\right)} .
\end{array}
$$

El Hamiltoniano resultante de esta transformación es de la forma

$$
\mathcal{H}=\mathcal{H}_{2}+\mathcal{H}_{3}+\ldots
$$

donde el término $\mathcal{H}_{k}$ es un polinomio homogéneo de grado $k$ en $u, U, v, V$; en particular $\mathcal{H}_{2}$ es (16).

El paréntesis de Poisson de cualquier función $F(u, v, U, V)$ con $\mathcal{H}_{2}$ es

$$
\left\{F ; \mathcal{H}_{2}\right\}=i \omega_{1}\left(u \frac{\partial F}{\partial u}-U \frac{\partial F}{\partial U}\right)+i \omega_{2}\left(v \frac{\partial F}{\partial v}-V \frac{\partial F}{\partial V}\right)
$$

Por tanto la derivada de Lie en el campo Hamiltoniano determinado por $\mathcal{H}_{2}$ es el operador

$$
L_{0}=i \omega_{1}\left(u \frac{\partial}{\partial u}-U \frac{\partial}{\partial U}\right)+i \omega_{2}\left(v \frac{\partial}{\partial v}-V \frac{\partial}{\partial V}\right) .
$$

En el álgebra compleja $\mathcal{A}$ de polinomios en $(u, v, U, V)$,

$$
L_{0}\left(u^{m} U^{n} v^{p} V^{q}\right)=\left[i \omega_{1}(m-n)+i \omega_{2}(p-q)\right] u^{m} \cdot U^{n} v^{p} V^{q}
$$

en otras palabras, el monomio $u^{m} U^{n} v^{p} V^{q}$ es el vector propio de $L_{0}$ para el valor propio $i \omega_{1}(m-n)+i \omega_{2}(p-q)$. Además, en ausencia de resonancias entre $\omega_{1}$ y $\omega_{2}$, el núcleo de $L_{0}$ en el álgebra $\mathcal{A}$ está generado por los monomios $(u U)^{m}(v V)^{p}$.

De acuerdo con Deprit [4], la normalización de $\mathcal{H}$ puede extenderse por medio de una transformación canónica $(u, v, U, V) \longrightarrow\left(u^{\prime}, v^{\prime}, U^{\prime}, V^{\prime}\right)$ cuyo efecto será transformar cada término $\mathcal{H}_{k}$ en un elemento $\mathcal{K}_{k}$ del núcleo de $L_{0}$, es decir, en un polinomio en $(u U)^{m}$ y $(v V)^{p}$ solamente. De aquí se seguirá, en particular, que, para todo $k>0, \mathcal{K}_{2 k+1}$ será cero.

Una vez efectuada la normalización, aplicamos la transformación completamente canónica de Poincaré

$$
\begin{array}{ll}
u^{\prime}=\sqrt{\Phi} e^{i \phi}, & v^{\prime}=\sqrt{\Psi} e^{-i \psi}, \\
U^{\prime}=-i \sqrt{\Phi} e^{-i \phi}, & V^{\prime}=i \sqrt{\Psi} e^{i \psi} .
\end{array}
$$


de variables complejas a las de ángulo-acción. El Hamiltoniano resultante es

$$
\mathcal{K}=\omega_{1} \Phi-\omega_{2} \Psi+A \Phi^{2}-2 B \Phi \Psi+C \Psi^{2}+\ldots,
$$

siendo los coefientes $A, B$ y $C$ funciones de los parámetros estructurales.

A hora estamos en condiciones de aplicar el teorema de Arnold [1, 10]. Los equilibrios $E_{2}$ y $E_{4}$ serán estables si la cantidad

$$
D_{4}=A \omega_{2}^{2}-2 B \omega_{1} \omega_{2}+C \omega_{1}^{2} .
$$

es distinta de cero; de acuerdo con Meyer y Laub [10], si fuese $D_{4}=0$ se hace necesario ir hasta el grado 6 y así sucesivamente. En el caso de la Tierra no es necesario.

Como función de $\omega_{1}$ y $\omega_{2}, D_{4}$ adopta la forma

$$
D_{4}=(M-R P) / Q
$$

donde las partes $Q, R, M$ y $P$ son

$$
\begin{aligned}
Q & =48 \omega_{1}^{4} \omega_{2}^{4}\left(\omega_{1}^{2}-4 \omega_{2}^{2}\right)\left(4 \omega_{1}^{2}-\omega_{2}^{2}\right)\left(\omega_{1}^{2}-\omega_{2}^{2}\right)^{2} r_{2}^{2} \\
R^{2} & =\left(\omega_{1}^{2}-\omega_{2}^{2}\right)^{2}+8 \omega^{2}\left(2 \omega^{2}-\omega_{1}^{2}-\omega_{2}^{2}\right), \\
M & =\sum_{0 \leq \ell \leq 5} \sum_{\substack{0 \leq m \leq n \leq 7 \\
m+n=9-\ell}} M_{\ell, m, n} \omega^{2 \ell}\left(\omega_{1}^{2 m} \omega_{2}^{2 n}+\omega_{1}^{2 n} \omega_{2}^{2 m}\right), \\
P & =\sum_{0 \leq \ell \leq 4} \sum_{\substack{0 \leq m \leq n \leq 6 \\
m+n=8-\ell}} P_{\ell, m, n} \omega^{2 \ell}\left(\omega_{1}^{2 m} \omega_{2}^{2 n}+\omega_{1}^{2 n} \omega_{2}^{2 m}\right) .
\end{aligned}
$$

El denominador $Q$ es fácil de obtener; en contraste, el numerador de $D_{4}$ requiere laboriosas manipulaciones. No queremos entrar en los detalles de las operaciones. Pero puede haber algunos lectores que intenten recrearse comprobando nuestros resultados. Para ayudarles, reproducimos en la Tabla 4 los coeficientes de $M$ y $P$ que hemos obtenido. El texto impreso es, en sí mismo, el resultado de transcripciones electrónicas, por lo que el riesgo de cometer errores de impresión es prácticamente nulo.

Los polinomios $M$ y $P$ y la función irracional $R$ son simétricos en $\omega_{1}$ y $\omega_{2}$. El denominador $Q$ no lo es, porque $r_{2}$ no es una función simétrica 


\begin{tabular}{rr}
\hline \\
$M_{0,2,7}=$ & -212 \\
$M_{0,3,6}=$ & 1086 \\
$M_{0,4,5}$ & -874 \\
$M_{1,1,7}=$ & 840 \\
$M_{1,2,6}=$ & -5842 \\
$M_{1,3,5}=$ & -9500 \\
$M_{1,4,4}=$ & 1542 \\
$M_{2,0,7}=$ & -4500 \\
$M_{2,1,6}=$ & -195 \\
$M_{2,2,5}=$ & 140310 \\
$M_{2,3,4}=$ & 161889 \\
$M_{3,0,6}=$ & 54000 \\
$M_{3,1,5}=$ & -32460 \\
$M_{3,2,4}=$ & -1158780 \\
$M_{3,3,3}=$ & -889920 \\
$M_{4,0,5}=$ & -216000 \\
$M_{4,1,4}=$ & 270000 \\
$M_{4,2,3}=$ & 4093200 \\
$M_{5,0,4}=$ & 288000 \\
$M_{5,1,3}=$ & -763200 \\
$M_{5,2,2}=$ & -1987200 \\
$M_{0}$
\end{tabular}$\quad \quad \quad\left\{\begin{array}{lr}P_{0,2,6}= & 116 \\
P_{0,3,5}= & -770 \\
P_{0,4,4}= & 654 \\
P_{1,1,6}= & -840 \\
P_{1,2,5}= & 5850 \\
P_{1,3,4}= & 12414 \\
P_{2,0,6}= & 4500 \\
P_{2,1,5}= & 1335 \\
P_{2,2,4}= & -122295 \\
P_{2,3,3}= & -102780 \\
P_{3,0,5}= & -36000 \\
P_{3,1,4}= & 37800 \\
P_{3,2,3}= & 727200 \\
P_{4,0,4}= & 72000 \\
P_{4,1,3}= & -190800 \\
P_{4,2,2}= & -496800 \\
\end{array}\right.$

Tabla 1: Coeficientes de los polinomios $M$ y $P$ en $D_{4}$ en función de $\omega_{1}$ y $\omega_{2}$.

en $\omega_{1}$ y $\omega_{2}$. Hemos excluido las resonancias entre $\omega_{1}$ y $\omega_{2}$, por tanto, la división por $Q$ no representa ningún problema.

Para comprobar los cálculos, hemos calculado el elemento crítico $D_{4}$ de otra forma, esta vez como una función racional en los parámetros estructurales $\alpha$ y $\beta$. Tediosas simplificaciones, ejecutadas también de manera electrónica, condujeron a la fórmula

$$
D_{4}=\left(\frac{N}{24(1-\beta)^{2} D}\right)\left(\frac{\omega^{2}}{r_{2}^{2}}\right) .
$$




\begin{tabular}{|c|c|c|c|c|}
\hline$A_{0,0}=$ & -57816 & $A_{1,0}=191232$ & $A_{2,0}=$ & 31175 \\
\hline$A_{0,1}=$ & 130032 & $A_{1,1}=105514$ & $A_{2,1}=$ & -92485 \\
\hline$A_{0,2}=$ & 188207 & $A_{1,2}=-60780$ & $A_{2,2}=$ & -195053 \\
\hline$A_{0,3}=$ & 88807 & $A_{1,3}=-93886$ & $A_{2,3}=$ & 7153 \\
\hline$A_{0,4}=$ & 15504 & $A_{1,4}=-18724$ & $A_{2,4}=$ & 5608 \\
\hline$A_{0,5}=$ & 346 & $A_{1,5}=-1956$ & $A_{2,5}=$ & 602 \\
\hline$A_{0,6}=$ & -532 & $A_{1,6}=$ & & \\
\hline$A_{0,7}=$ & -48 & & & \\
\hline$A_{3,0}=$ & -80454 & $A_{4,0}=-74745$ & $A_{5,0}=$ & -5504 \\
\hline$A_{3,1}=$ & -186420 & $A_{4,1}=35711$ & $A_{5,1}=$ & 1168 \\
\hline$A_{3,2}=$ & 77946 & $A_{4,2}=-11452$ & $A_{5,2}=$ & -164 \\
\hline$A_{3,3}=$ & 1404 & $A_{4,3}=$ & & \\
\hline$A_{3,4}=$ & -1476 & & & \\
\hline
\end{tabular}

Tabla 2: Coeficientes del polinomio $N$ en la expresión de $D_{4}$ en función de los coeficientes estructurales $\alpha$ y $\beta$.

En el factor adimensional, $N$ y $D$ son los polinomios

$$
\begin{gathered}
N=\sum_{0 \leq m \leq 5} \sum_{0 \leq n \leq 7-m} A_{m, n} \alpha^{m} \beta^{n} ; \\
D=\left[(\alpha-\beta)^{2}+8(\alpha+\beta)\right]\left[4(\alpha-\beta)^{2}-9(1+\alpha \beta)+41(\alpha+\beta)\right],
\end{gathered}
$$

con los coeficientes $A_{m, n}$ que se expresan en la Tabla 2

Con los valores de $\Gamma_{20}$ y $\Gamma_{22}$ de (15) y escogiendo las unidades de longitud y tiempo de forma que sea

$$
\mu=1, \quad \omega=1 \Rightarrow r_{0}=1,
$$

tanto si usamos (18) como (17) encontramos que $D_{4}=1.49991$ para la Tierra. En consecuencia, de acuerdo con el teorema de Arnold, los equilibrios $E_{2}$ y $E_{4}$ son estables en el sentido de Liapunov.

El valor numérico de $C_{20}$ es conocido con una gran exactitud, pero no así el de $C_{22}$. Por tanto habría que preguntarse en que entorno de 


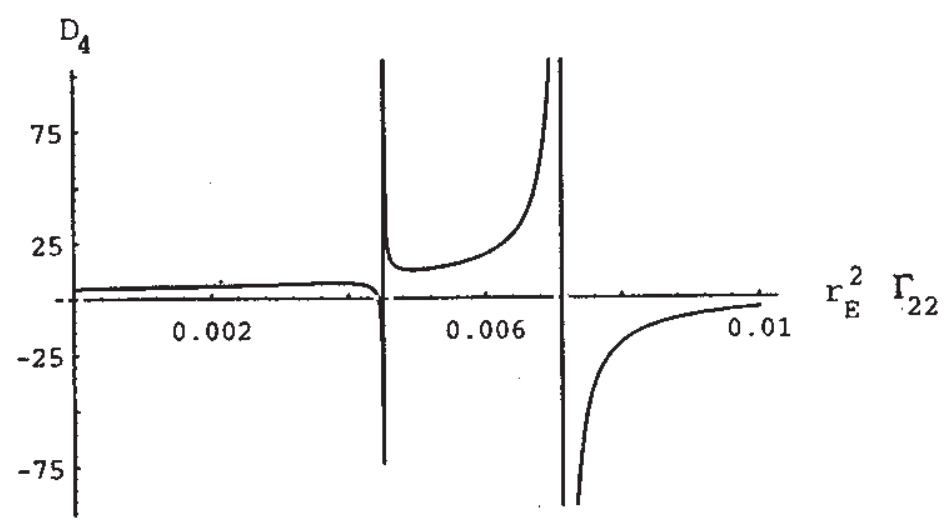

Figura 2: Valores de $D_{4}$ en función de $\Gamma_{22}$, para el valor de $\Gamma_{20}$ correspondiente a la Tierra. Las unidades de longitud y tiempo se han escogido de forma que es $\mu=1$ y $\omega=1$. La primera discontinuidad corresponde a la resonancia 2:1 y la segunda a la 1:1. A la derecha de esta última los equilibrios $E_{2}$ y $E_{4}$ son inestables.

$\Gamma_{22}$ se mantiene la estabilidad. La respuesta a esta cuestión se da en la Figura 2. Allí hemos dibujado $D_{4}$ como una función de $\Gamma_{22}$, para los valores de $\oplus$ y $\Gamma_{20}$ correspondientes a la Tierra. Para el valor $\oplus^{2} \Gamma_{22}=$ $4.3862 \times 10^{-3}\left(\Gamma_{22}=0.192385\right)$ se anula $D_{4}$, con lo que para asegurar la estabilidad tendríamos que ir hasta el grado 6 . El primer punto de discontinuidad de la curva corresponde a la resonancia $2: 1$, mientras que el segundo corresponde a la resonancia 1:1. Este último punto es el límite de estabilidad lineal; a la derecha de él los valores propios tienen parte real no nula. En el caso de resonancia 2:1 no es de aplicación el teorema de Arnold, pues la normalización nos lleva a un Hamiltoniano cuyos órdenes impares no son idénticamente nulos.

\section{Conclusiones}

El teorema de Arnold es una poderosa herramienta para decidir sobre la estabilidad de un equilibrio en un sistema dinámico con sólo dos grados de libertad, cuando el Hamiltoniano en el equilibrio es una forma cuadrática no definida. Hasta ahora, el teorema había tenido solamente 
aplicación en astronomía, en los equilibrios triangulares del problema restringido plano de tres cuerpos. Con este artículo, añadimos otra aplicación.

Llegamos a conclusiones definitivas acerca de la estabilidad orbital de satélites de comunicaciones de la Tierra. Sin embargo, en el desarrollo de la demostración, mantenemos todos los parámetros que intervienen en el problema como símbolos y los manipulamos como símbolos, no como números. Por esta razón, el desarrollo puede aplicarse a otros planetas.

Un desarrollo completamente simbólico no es una tarea para ser ejecutada a mano; es demasiado compleja. Por ello, hemos organizado los cálculos como un conjunto de programas a ejecutar automáticamente por ordenadores con un procesador científico de textos matemáticos, como Mathematica. En cierto sentido, podemos decir de verdad que hemos automatizado la aplicación del teorema de Arnold.

\section{Agradecimientos}

El segundo autor está en deuda con el Teniente de Navio Martín Lara Coira por las frecuentes discusiones sobre el tema de este artículo. Ambos autores agradecen gustosamente el apoyo técnico que les dió tan generosamente el Grupo de Mecánica Espacial en la Universidad de Zaragoza, en particular, al Profesor Antonio Elipe Sánchez.

Este artículo es un extracto de la tesis doctoral que Teodoro López Moratalla presentará en la Universidad de Zaragoza para obtener el grado de doctor. 


\section{Apéndice \\ Programa para una normalización}

Este programa en Mathematica efectúa la normalización de un Hamiltoniano perteneciente al álgebra de polinomios en variables complejas, cuyo orden cero es de la forma (16).

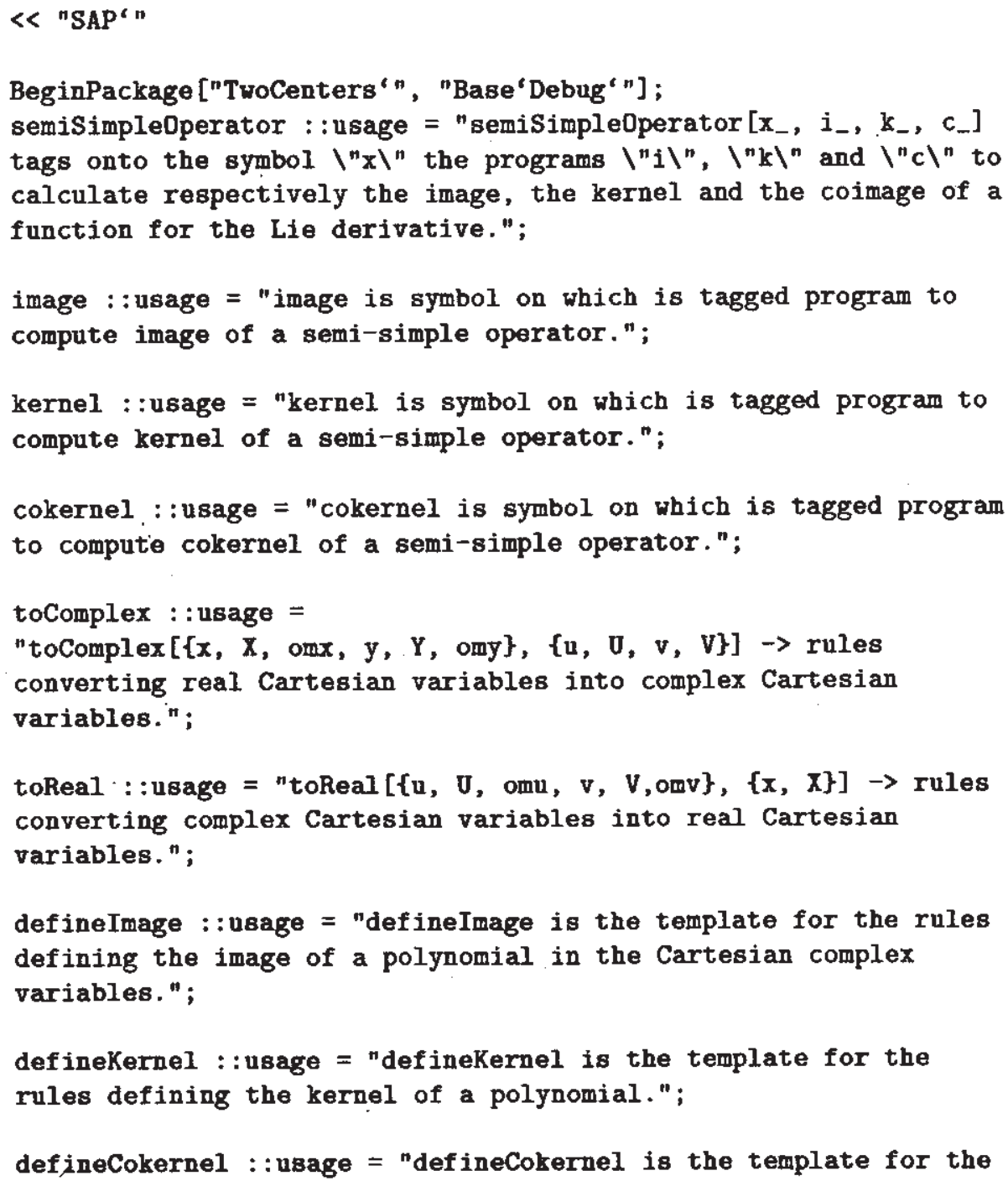




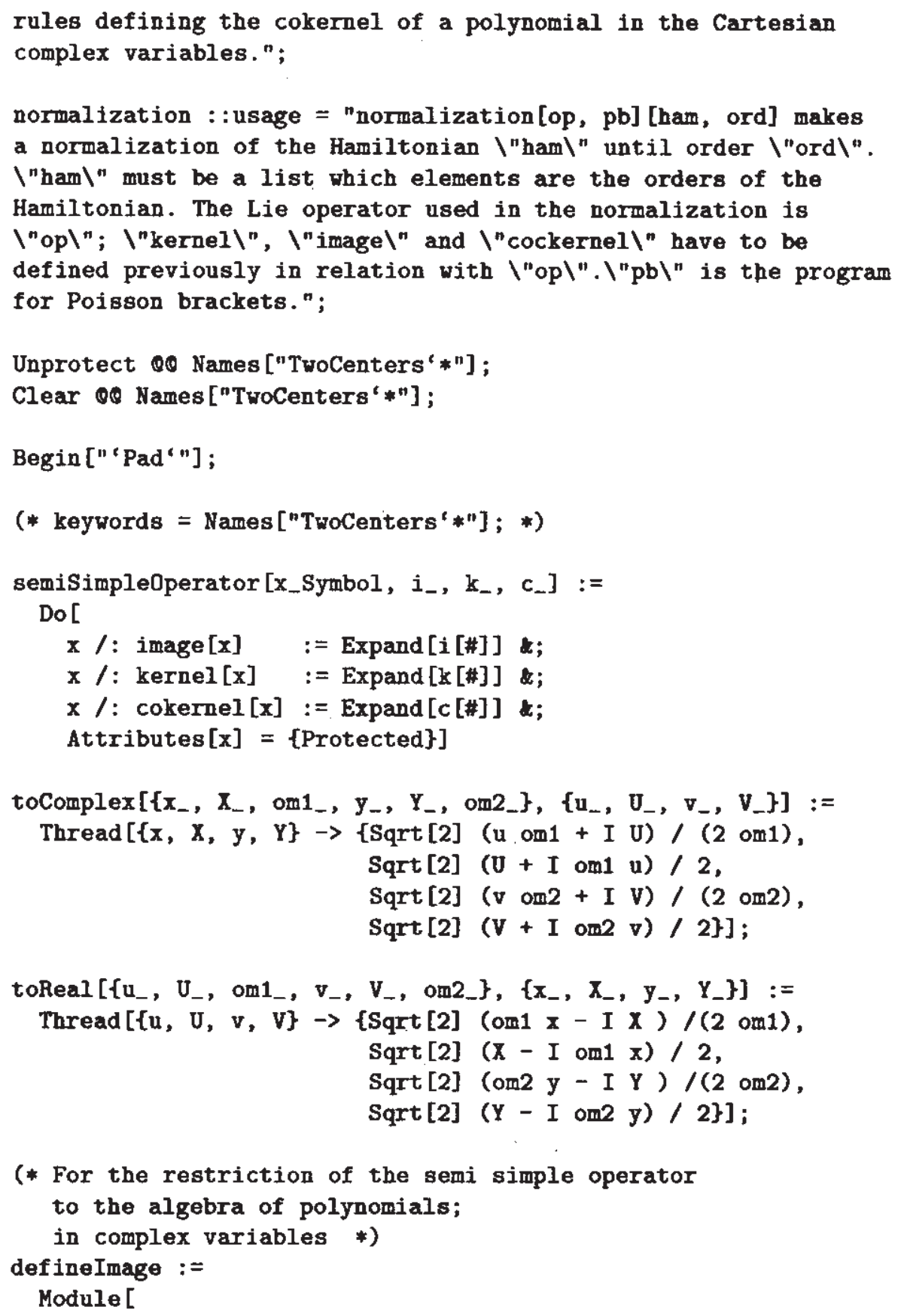

(* For the restriction of the semi simple operator to the algebra of polynomials; 


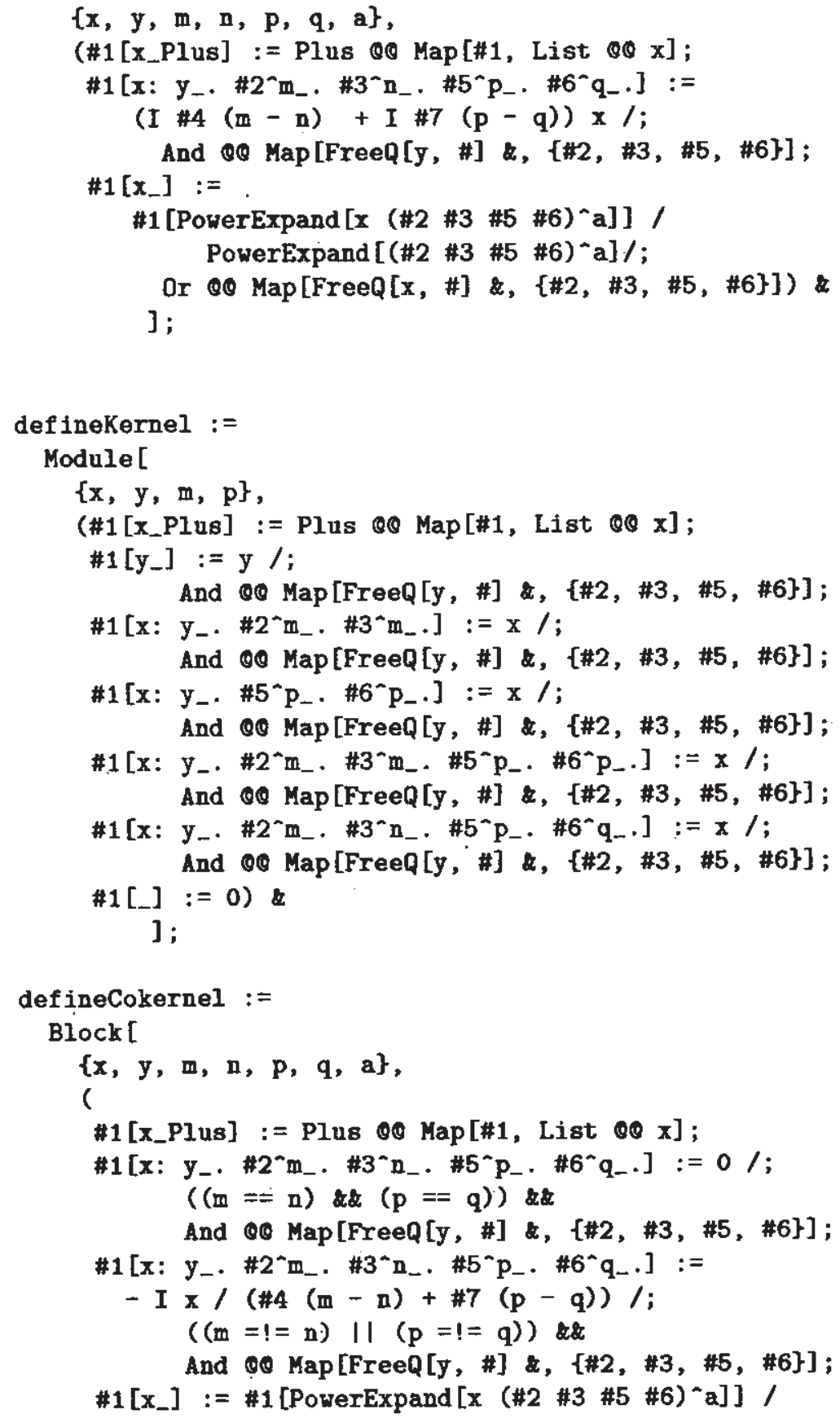




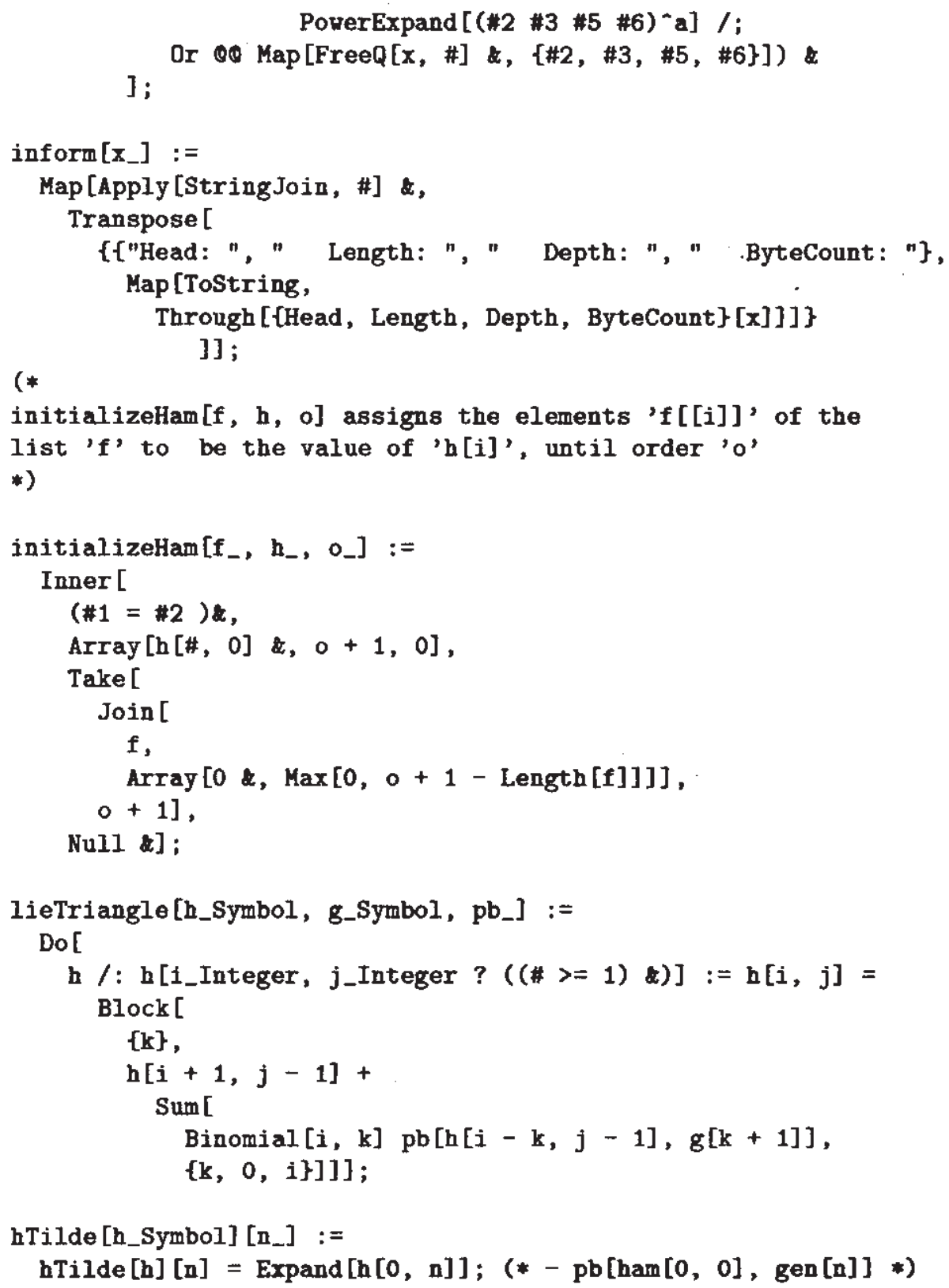




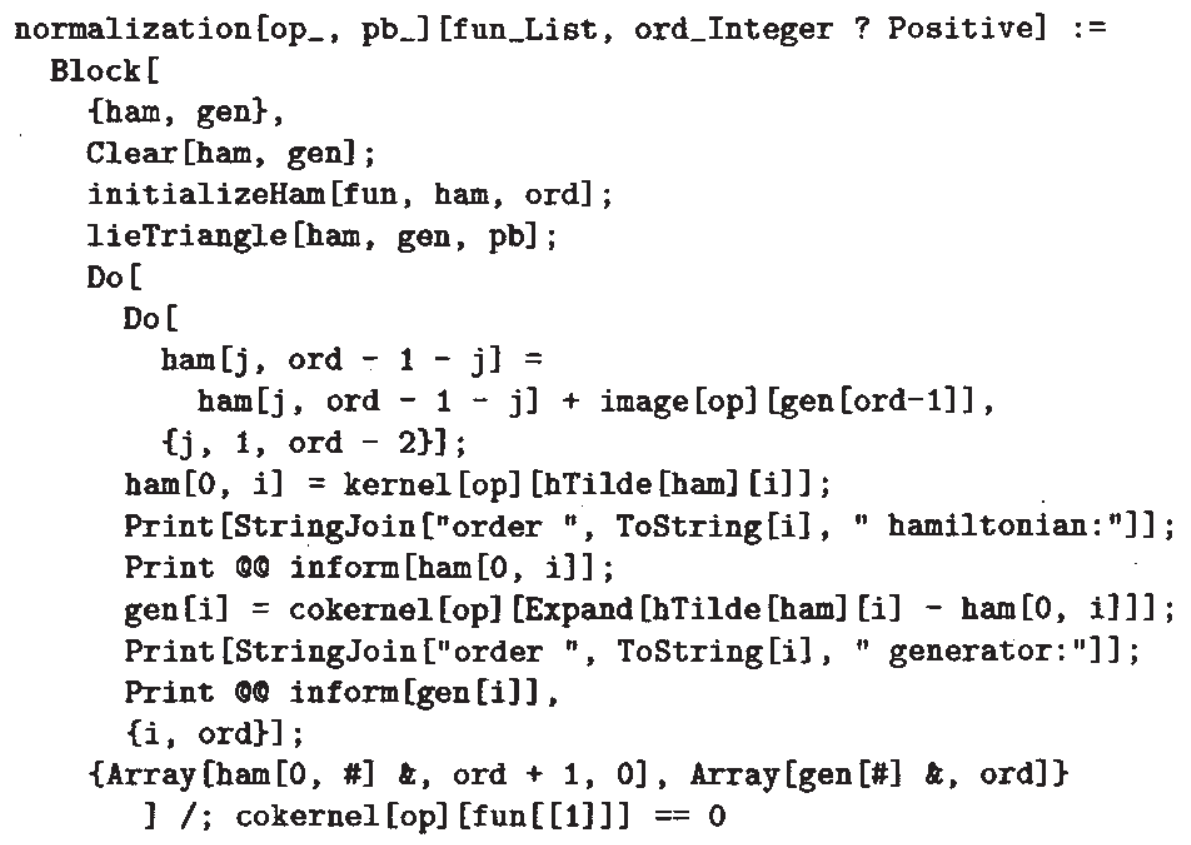

End [ ] ;

Protect 00 Names ["TwoCenters"*n];

EndPackage [ ];

\section{Referencias}

[1] Arnold, V.I.: The stability of the equilibrium position of a Hamiltonian system of ordinary differential equations in the general elliptic case. Soviet Math. Dokl. 2 (1961), 247-249.

[2] Blitzer, L., Boughton, E.M., Kang, G. y Page, R.M.: Effect of ellipticity of the equator on 24-hour nearly circular satellite orbits. J. Gephys. Res. 67 (1962), 329-335.

[3] Deprit, A.: Motion in the Vicinity of the Triangular Libration Centers. Lectures in Applied Mathematics (American Mathematical 
Society) 6 (1966), Space Mathematics. Part II, 1-30.

[4] Deprit, A.: Canonical Transformations Depending on a Small Parameter. Celes. Mech. 1 (1969), 12-30.

[5] Deprit, A. y Deprit, E.: Implicit Equations Solved by Lie Transformations. Remitido a Celes. Mech. en 1995.

[6] Deprit, A. y Deprit-Bartholomé, A.: Stability of the Triangular Lagrangian Points. Astron. J. 72 (1967), 173-179.

[7] Heiskanen, W.A. y Moritz, H.: Geodésia Física. (Inst. Geog. Nac. y Inst. Astronom. y Geod., Madrid, 1985).

[8] Howard, J.: Spectral Stability of Relative Equilibria. Celes. Mech. 48 (1990), 267-288.

[9] Laub, A. y Meyer, K.: Canonical Forms for Symplectic and Hamiltonian Matrices. Celes. Mech. 9 (1974), 213-238.

[10] Meyer, K. y Schmidt, D.: The Stability of the Lagrange Triangular Point and a Theorem of Arnold. J. Differential Equations 62 (1986), 222-236.

[11] Morando, B.: Orbites de résonance des satellites de 24 heures. Bulletin Astronomique 24 (1963), 47-67.

[12] Musen, P. y Bailie, A.E.: On the Motion of a 24-Hour Satellite. J. Gephys. Res. 67 (1962), 1123-1132.

[13] Wolfram, S.: Mathematica, a System for Doing Mathematics by Computer. (Adison Wesley, 1993).

National Institute of Standards and Technology

Gaithersburg, MD 20899

U.S.A.

e-mail: andre.deprit@nist.gov

Real Instituto y Observatorio

de la Armada

11110 San Fernando

SPAIN

e-mail: ccgeneral@czv1.uca.es Recibido: 31 de Mayo de, 1995 